\title{
Model Predictive Current Control Strategy with Reduced Computation Burden
}

\author{
Jie Yuan, Dingdou Wen, and Yang Zhang $\mathbb{D}$ \\ College of Electrical and Information Engineering, Hunan University of Technology, Zhuzhou 412007, China \\ Correspondence should be addressed to Yang Zhang; hut_zy@163.com
}

Received 23 November 2020; Revised 31 December 2020; Accepted 19 January 2021; Published 5 February 2021

Academic Editor: Xiaodong Sun

Copyright ( 2021 Jie Yuan et al. This is an open access article distributed under the Creative Commons Attribution License, which permits unrestricted use, distribution, and reproduction in any medium, provided the original work is properly cited.

\begin{abstract}
In this paper, three model predictive current control (MPCC) schemes for permanent magnet synchronous motors (PMSM) are studied. The first control scheme is the traditional optimal duty cycle model predictive current control (ODC-MPCC). In this scheme, according to the principle of minimizing the cost function, the optimal voltage vector is selected from the six basic voltage vectors which are optimized simultaneously with the duty, and then, the optimal voltage vector and its duty are applied to the inverter. In order to reduce the computational burden of ODC-MPCC, a second control scheme is proposed. This scheme optimizes the voltage vector control set, reducing the number of candidate voltage vectors from 6 to 2 . Finally, according to the principle of minimizing the cost function, the optimal voltage vector is found from the two voltage vectors, and the optimal voltage vector and its duty cycle are applied to the inverter. In addition, in order to further improve the steady-state performance, another vector selection method is introduced. In the combination of voltage vectors, the third control scheme extends the combination of voltage vectors in the second control scheme. The simulation results show that the second control scheme not only reduces the computational burden of the first control scheme but also obtains steady-state performance and dynamic performance equivalent to the first control scheme. The third control scheme obtains better steady-state performance without significantly increasing the computational burden and has dynamic performance comparable to the first and second control schemes.
\end{abstract}

\section{Introduction}

Currently, the new energy vehicle industry is undergoing rapid development. Electric vehicles (EVs) not only require frequent start and stop under working conditions but also require good steady-state performance at high speeds. The PMSM not only has the characteristics of small size, lightweight, good reliability, and large torque when running at medium and low speeds, it can also carry out weak magnetic acceleration and low noise $[1,2]$. Therefore, the PMSM is the best choice as the drive motor for EVs. The control performance and reliability of PMSM will not only affect the working status of related equipment but also affect the economic benefits of the entire industry. Therefore, it is particularly important to study the high-performance control strategy driven by PMSM [3].

According to the different input control strategies of PMSM, it can be roughly divided into two categories [4]: continuous and discrete. The typical representatives of continuous input control strategies are vector control (VC) and continuous control set model predictive control (CCS-MPC) $[5,6]$. This input control strategy is more prominent in robustness, stability, or adaptability. Discrete input control strategies include direct torque control (DTC) [7] and finite-control-set model predictive control (FCS-MPC) [8,9]. This type of control strategy directly considers the discrete switching state of the inverter [10], so it usually does not require a modulation module and has better dynamic response capabilities. Aiming at the requirements of EVs with high precision and rapid response, the FCS-MPC is considered in this paper.

From the perspective of control objectives, the hot research areas of PMSM FCS-MPC include model predictive torque control (MPTC) $[11,12]$ and model predictive current control (MPCC) [13]. MPTC is usually implemented 
by selecting a voltage vector that can minimize the cost function. The cost function includes torque and flux linkage. When implemented with a digital signal processor, the motor torque and magnetic flux in the stator core are not easy to obtain directly and need to be estimated with the stator phase current measured by the motor, which requires more calculation time. In MPTC, the selection of weighting factors is also problematic. There is no general theory to guide the design of weighting coefficients. In specific applications, repeated simulations and experiments are generally used to optimize the coefficients [14], which hinders the further development and practicability of the MPTC method to a certain extent. The MPCC that uses the stator current as the control variable does not need to estimate torque and magnetic flux, so it is easy to implement and has a small computational burden. In summary, for PMSM drives, MPCC is a simpler solution than MPTC [15].

According to the different number of voltage vectors employs during one control period, MPC can be divided into single vector MPC, double vector MPC, and triple vector MPC. The single number of voltage vectors selected is the cause of the large current pulsation of single vector MPC [16]. Therefore, the research on double vector MPC and triple vector MPC is more important. In dual-vector MPC, the main research goal is generally to improve the steadystate performance of the system. Duty cycle and voltage vector are optimized at the same time to improve the steadystate performance of the system in Ref. [17]. Since the second voltage vector is fixed to a zero vector, the steady-state is poor at high speed. Two groups of basic voltage vectors are combined arbitrarily, and a group of optimal voltage vector combinations is selected among all kinds of combinations [18]. The abundant voltage vector combination makes the voltage vector have a wider selection range, and the steadystate performance is improved; at the same time, the computational burden is increased. However, this is not what we want to see. In Ref. [19], after selecting the first optimal voltage vector, the optimal voltage vector combination is selected from the seven combinations of the basic voltage vector and the first optimal voltage vector. In order to obtain better steady-state performance and reduce the computational burden of the algorithm, literature [20] by calculating the q-axis current slopes for different voltage vector and finding the optimal intersection point. Although the system has achieved better steady-state than the traditional dual-vector MPCC performance, its computational burden is still relatively large. The position of the reference voltage vector is determined by deadbeat principle, and the voltage vector nearest to the reference voltage vector is selected as the optimal voltage vector, and the duration is calculated according to the deadbeat principle [21]. This method requires only one current prediction, and it has achieved a better steady-state performance than traditional deadbeat current control. In Ref. [22], an MPCC based on the current track circle is proposed. By establishing a reference coordinate system, this strategy omits the design of the cost function and reduces the computational burden of the algorithm, but it is essentially the same as the deadbeat control method used in Ref. [21]. There is no doubt that these works have made certain contributions to the study of dual-vector MPCC.

Aiming at the problem that the optimal duty cycle control (ODC-MPCC) is performed 6 times in total, the calculation burden of current prediction is heavy. This paper aims to reduce the computational burden of the control algorithm. The method of selecting the optimal voltage vector for deadbeat q-axis current control and the method of selecting the optimal voltage vector for deadbeat current control are run in the same simulation model. When selecting voltage vectors, the number of candidate voltage vectors can be reduced from 6 to 2, because the optimal voltage vectors selected by deadbeat $q$-axis current control and deadbeat current control are in the same sector and adjacent. Therefore, MPCC with reduces computational burden (RCBI-MPCC) is proposed. The algorithm uses the idea of deadbeat current control, but essentially it is $i_{q}$ deadbeat control. The algorithm combines the null vector and the two basic voltage vectors in the sector, respectively, and determines the duration of the voltage vector based on the principle of deadbeat $q$-axis current. Finally, the voltage vector that minimizes the cost function is selected as the optimal voltage vector. In addition, in order to further improve the steady-state performance of the system, the algorithm is improved on the basis of not significantly increasing the computational burden, and a new MPCC with reduces computational burden (RCBII-MPCC) is proposed. When performing voltage vector combination, expand the selection range of the second voltage vector to the candidate voltage vector and null voltage vector in the sector. Finally, the steady-state and dynamic control effects of ODC-MPCC and the method proposed in this paper are compared and analyzed through simulation, and the feasibility and effectiveness of the proposed method are verified.

The main contributions of this paper are as follows:

(1) It is found that the optimal voltage vector selected by $i_{q}$ deadbeat and the optimal voltage vector selected by current deadbeat are in the same sector and adjacent to each other.

(2) Compared with the ODC-MPCC, the proposed MPCC not only reduces the computational burden but also improves the steady-state performance of the system.

(3) Compared with the ODC-MPCC, the proposed MPCC does not change the dynamic performance of the system.

\section{Discrete Mathematical Model of PMSM}

The voltage equation of surface-mounted PMSM in the synchronous $d-q$ reference frame is as follows:

$$
\begin{aligned}
& u_{q}=R_{s} i_{q}+L_{s} \frac{\mathrm{d} i_{q}}{\mathrm{~d} t}+\omega_{\mathrm{re}} L_{s} i_{d}+\omega_{\mathrm{re}} \psi_{f}, \\
& u_{d}=R_{s} i_{d}+L_{s} \frac{\mathrm{d} i_{d}}{\mathrm{~d} t}-\omega_{\mathrm{re}} L_{s} i_{q},
\end{aligned}
$$


where $u_{d}$ and $u_{q}$ are the $d$-axis and $q$-axis components of the stator voltage. $i_{d}$ and $i_{q}$ are the $d$-axis and $q$-axis stator current. $R_{s}$ is the stator resistance. $L_{s}$ is the stator inductance. $\omega_{\text {re }}$ is the rotor electrical velocity. $\psi_{f}$ is rotor flux established by the permanent magnets.

The motor current is selected as the state variable, and the motor voltage equation can be written as the state equation as follows:

$$
\dot{i}=A i+B u+D,
$$

where $\quad A=\left[\begin{array}{cc}-(R / L) & \omega_{\mathrm{re}} \\ -\omega_{\mathrm{re}} & -(R / L)\end{array}\right], \quad B=\left[\begin{array}{cc}(1 / L) & 0 \\ 0 & (1 / L)\end{array}\right]$, $D=\left[\begin{array}{c}0 \\ -\left(\omega_{\mathrm{re}} \psi_{f} / L\right)\end{array}\right]$, and $i=\left[\begin{array}{ll}i_{d} & i_{q}\end{array}\right]^{T}$.

Formula (3) is processed by the first-order Euler method discretization as follows:

$$
\stackrel{g}{i}=\frac{i(k+1)-i(k)}{T_{s}},
$$

where $T_{s}$ is the sampling period.

Substituting formula (4) into formula (3), the PMSM prediction model is obtained as follows:

$$
i(k+1)=A^{\prime}(k) i(k)+B^{\prime}(k) u(k)+D^{\prime}(k),
$$

where $\quad i(k)=\left[i_{d}(k) i_{q}(k)\right], \quad u(k)=\left[\begin{array}{ll}u_{d}(k) & u_{q}(k)\end{array}\right]$, $A^{\prime}(k)=\left[\begin{array}{cc}1-\left(T_{s} R / L\right) & T_{s} \omega_{\text {re }}(k) \\ -T_{s} \omega_{\text {re }}(k) & 1-\left(T_{s} R / L\right)\end{array}\right], \quad B^{\prime}(k)=$ $\left[\begin{array}{cc}T_{s} / L & 0 \\ 0 & T_{s} / L\end{array}\right]$, and $D^{\prime}(k)=\left[\begin{array}{c}0 \\ -\left(T_{s} \psi_{s} / L\right) \omega_{\mathrm{re}}(k)\end{array}\right] \cdot i_{d}(k)$ and $i_{q}(k)$ are $d$-and $q$-axis state current at $k$ th sampling instant. $i$ $(k+1)$ is state current at $(k+1)$ th sampling instant. $T_{s}$ is the sampling period. $u_{d}(k)$ and $u_{q}(k)$ are $d$-and $q$-axis state voltage at $k$ th sampling instant. $\omega_{\text {re }}(k)$ is the state rotor electrical velocity at $k$ th sampling instant.

\section{Traditional Optimal Duty Cycle MPCC}

According to the principle of q-axis current deadbeat control, the method for ODC-MPCC to select the best voltage vector is

The principle of $i_{q}$ deadbeat control is as follows:

$$
i_{q}(k+1)=i_{q}(k)+s_{i} \alpha_{i} T_{s}+s_{0}\left(T_{s}-\alpha_{i} T_{s}\right)=i_{q}^{*},
$$

where $i_{q}(k), i_{q}(k+1)$, and $i_{q}^{*}$ are the feedback value, predicted value, and reference value of $q$-axis current. $\alpha_{i}$ is the duty cycle of the active-voltage vector.

The duty cycle of the active-voltage vector can be obtained by formula (6) as follows:

$$
\alpha_{i}=\frac{i_{q}^{*}-i_{q}(k)-s_{0} T_{s}}{T_{s}\left(s_{i}-s_{0}\right)},
$$

where $s_{i}$ and $s_{0}$ are the $q$-axis current slopes when the activevoltage vector and null voltage vector are applied, respectively, $i=1,2, \ldots, 6$. It should be noted that the duty cycle is limited to the range of $[0,1]$ in practical applications.

$$
\begin{gathered}
s_{0}=\frac{\mathrm{d} i_{q}}{\mathrm{~d} t} \mid u_{q}=0, \\
s_{i}=\frac{\mathrm{d} i_{q}}{\mathrm{~d} t} \mid u_{q}=u_{q i} .
\end{gathered}
$$

Simultaneous optimization of voltage vector and duty cycle. The $u_{d}$ and $u_{q}$ in formula (5) can be written as

$$
\begin{aligned}
& u_{q}=a_{i} u_{q i}, \\
& u_{d}=a_{i} u_{d i},
\end{aligned}
$$

where $u_{q i}$ is the $q$-axis stator voltage corresponding to the voltage vector $v_{i}, u_{d i}$ is the $d$-axis stator voltage corresponding to the voltage vector $v_{i}$, and $i=1,2, \ldots, 6$.

The 6 nonzero voltage vectors which optimized simultaneously with the duty cycle are used to predict the value of the stator current in the next period. The optimal voltage vector is obtained by minimizing the cost function $g$ :

$$
g=\left|i_{q}^{*}-i_{q}(k+1)\right|+\left|i_{d}^{*}-i_{d}(k+1)\right|,
$$

where $g$ is the cost function. $i_{d}(k+1)$ and $i_{q}(k+1)$ are $d$ - and $q$-axis state current at $(k+1)$ th sampling instant. $i_{q}^{*}$ is the reference value of $q$-axis current.

\section{The Proposed MPCC}

4.1. MPCC with Reduced Computation Burden I. ODC-MPCC needs to find the optimal voltage vector from 6 basic voltage vectors, and its calculation burden is heavy. This paper analyzes the simulation results of selecting the optimal voltage vector in the same model for deadbeat $q$-axis current control and deadbeat current control, and a new method of selecting voltage vectors is proposed.

The method of selecting the voltage vector [14] using the deadbeat current control MPCC is

The reference voltage vector that should be applied at the next moment can be obtained by formulas (1) and (2) as follows:

$$
\begin{aligned}
& u_{d}(k+1)=L_{d} \frac{\left(i_{d}^{*}-i_{d}(k)\right)}{T_{s}}+R_{s} i_{d}^{*}-L_{q} i_{q}^{*} \omega_{r e}, \\
& u_{q}(k+1)=L_{q} \frac{\left(i_{q}^{*}-i_{q}(k)\right)}{T_{s}}+R_{s} i_{q}^{*}+L_{d} i_{d}^{*} \omega_{\mathrm{re}}+\psi_{f} \omega_{\mathrm{re}}
\end{aligned}
$$

where $u_{d}(k+1)$ and $u_{q}(k+1)$ are $d$ - and $q$-axis state voltage at $(k+1)$ th sampling instant. $L_{d}$ and $L_{q}$ are the $d$-axis and $q$ axis stator inductance.

The reference voltage vectors $u_{d}(k+1)$ and $u_{q}(k+1)$ are transformed to the $\alpha \beta$ frame. The phase angle of the reference voltage vector is obtained by formula (13). The sector where the reference voltage vector is located is determined by the phase angle of the reference voltage vector.

$$
\theta=\arctan \frac{u_{\beta}(k+1)}{u_{\alpha}(k+1)}
$$


where $u_{\alpha}(k+1)$ and $u_{\beta}(k+1)$ are the values of $u_{d}(k+1)$ and $u_{q}(k+1)$ after coordinate conversion.

If $u_{\beta}(k+1)<0$, then $\theta=\theta+2 \pi$.

The calculation method of the sector where the optimal voltage vector $u_{\text {opt }}$ is located is as follows:

$$
\sec =\text { floor }\left(\frac{3 \theta}{\pi}\right)+1,
$$

where sec represents the number of the sector, the floor is the function of rounding down. Table 1 shows sec and its corresponding voltage vector.

The method of selecting the optimal voltage vector for deadbeat current control and the method of selecting the optimal voltage vector for ODC-MPCC are both run in the same simulation model, and the sequence number difference relationship between the selected optimal voltage vectors under the two methods is obtained in the oscilloscope.

It can be seen from Figure 1 that there are three kinds of sequence number difference between the optimal voltage vector obtained by quadrature axis deadbeat current control and the optimal voltage vector obtained by deadbeat current control. The values are 0,1 , and, 5 respectively. 0 means that the optimal voltage vectors selected under the two methods are the same; 1 or 5 indicates that the optimal voltage vectors selected under the two methods are adjacent.

Read the serial numbers of two adjacent basic voltage vectors of sec, and formula (15) is used to explore the relationship between $u_{m}$ (the optimal voltage vector obtained by deadbeat current control) and $u_{n}$ (the optimal voltage vector obtained by ODC-MPCC). $u_{m}$ is located in the sector sec; judge whether $u_{n}$ is a voltage vector in the sector sec. And get the waveform diagram shown in Figure 2 in the oscilloscope.

$$
\text { error }=(n-\operatorname{sector}(\sec , 1))(n-\operatorname{sector}(\sec , 2)),
$$

where error is used to determine whether $n$ is equal to one of the numbers in the sector. $n$ is the sequence number of the optimal voltage vector obtained by the $i_{q}$ deadbeat method, and sec is the sector number. Sector $=[12 ; 23 ; 34 ; 45 ; 56 ; 6$ $1]$.

It can be seen from Figure 2 that the value of error is always 0 , indicating that $u_{n}$ has always been in the sector sec area. In combination with Figures 1 and 2, $u_{m}$ and $u_{n}$ are in the same sector and adjacent to each other. An MPCC with reduced computation burden I (RCBI-MPCC) is proposed. RCB I-MPCC only performs two current predictions.

The reference voltage vectors $u_{d}(k+1)$ and $u_{q}(k+1)$ that should be applied at the next moment are obtained by formulas (11) and (12). $u_{\alpha}(k+1)$ and $u_{\beta}(k+1)$ are obtained by coordinate transformation from the reference voltage vector.

The sector where the reference voltage vector is located is calculated by formula (13), and two effective voltage vectors that fall in the sector are selected as candidate voltage vectors. The duty cycle of the two candidate voltage vectors in the sector is calculated by formulas (7), and the optimal voltage vector combination that minimizes the cost function $g$ is selected. Compared with the traditional ODC-MPCC, RCBI-MPCC reduces the computational burden by $66.6 \%$.

4.2. MPCC with Reduced Computation Burden II. In terms of voltage vector selection, the RCBI-MPCC reduces the number of candidate voltage vectors to 2 , and there are only 2 combinations of selected voltage vectors. If the second voltage vector is not fixed to zero but selected between the zero vector and the effective voltage vector in the sector, the steady-state performance of the system will be improved. At this time, an MPCC with reduced computation burden II (RCBII-MPCC) is proposed. This method performs 3 current predictions, reduces the calculation burden by $50 \%$ compared with the traditional ODC-MPCC, and improves the steady-state performance. The implementation steps are as follows:

(1) The principle of deadbeat current control is used to predict the reference voltage vector that should be applied in the next cycle online and then convert the reference voltage vector to $\alpha \beta$ frame.

(2) The position angle is used to determine the sector where the reference voltage vector is located. Two effective voltage vectors in the sector are determined as the candidate voltage vector. The system obtains a total of three sets of candidate voltage vector combinations: the combination between two effective voltage vectors, the null voltage vector combined with two basic voltage vectors in the sector, respectively.

(3) The $i_{q}$ deadbeat control principle is used to calculate the duration of the effective voltage vectors are selected as the optimal voltage combination.

As shown in Figure 3, when the reference voltage vector falls in the first sector, the combination of candidate voltage vectors is $u_{1}$ and $u_{0} ; u_{2}$, and $u_{7} ; u_{1}$ and $u_{2}$.

The principle of deadbeat $q$-axis current control principle is used to allocate the duration of the selected voltage vectors $u_{i}$ and $u_{j}$.

When the combination of voltage vector is $u_{1}$ and $u_{2}$, assuming that the duration of $u_{1}$ is $t_{1}$, the formula (6) is changed as follows:

$$
\begin{aligned}
i_{q}(k+1) & =i_{q}(k)+s_{1} t_{1}+s_{2}\left(T_{s}-t_{1}\right)=i_{q}^{*}, \\
s_{1} & =s_{0}+\frac{u_{q_{1}}}{L_{s}},
\end{aligned}
$$

where $s_{1}$ and $s_{2}$ are the slopes of the q-axis when the voltage vectors $u_{1}$ and $u_{2}$ act, respectively.

The duration of $u_{1}$ can be calculated by formula (16) as follows:

$$
t_{1}=\frac{i_{q}^{*}-i_{q}(k)-s_{2} T_{s}}{s_{1}-s_{2}}
$$

When the combination of voltage vector is $u_{1}$ and $u_{0} ; u_{2}$ and $u_{7}$, formulas (5) and (6) are used to calculate the duty cycle of $u_{1}$ and $u_{2}$. The cost function values corresponding to 
TABLE 1: Effective voltage vector in sector sec.

\begin{tabular}{lc}
\hline Sec & Effective voltage vector \\
\hline 1 & $U_{1}, U_{2}$ \\
2 & $U_{2}, U_{3}$ \\
3 & $U_{3}, U_{4}$ \\
4 & $U_{4}, U_{5}$ \\
5 & $U_{5}, U_{6}$ \\
6 & $U_{6}, U_{1}$ \\
\hline
\end{tabular}

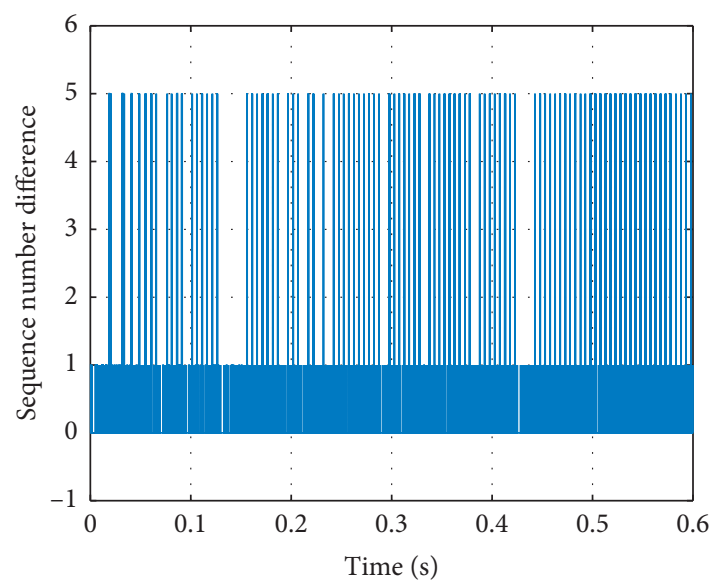

Figure 1: The sequence number difference between the optimal voltage vector selected by $i_{q}$ deadbeat control and the optimal voltage vector selected by $i_{d}$ and $i_{q}$ deadbeat control.

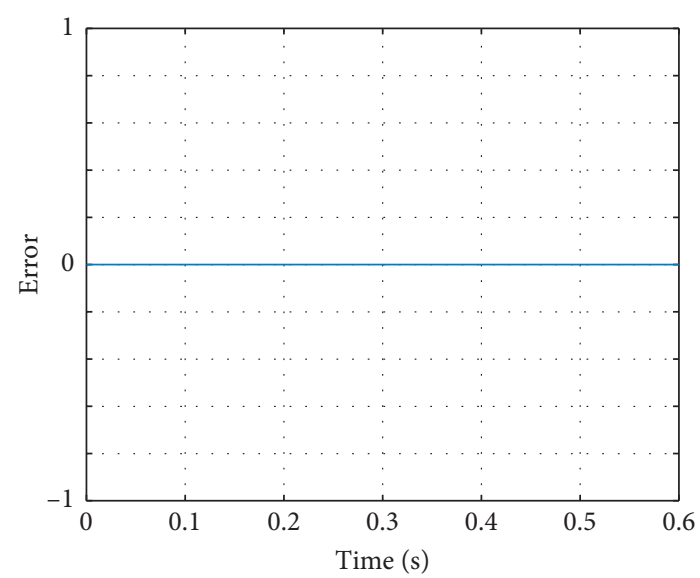

Figure 2: The sector difference of the optimal voltage vector selected by the two methods.

the above three voltage vector combinations are sorted and the optimal voltage vector combination is obtained.

The control strategy block diagram of this text is shown in Figure 4.

\section{Simulation Result Analysis}

The three double vector model predictive current control strategies mentioned above are simulated and verified in MATLAB/Simulink software. The parameters of PMSM are flux linkage $0.1 \mathrm{~Wb}$, stator inductance $1.625 \mathrm{mH}$, stator

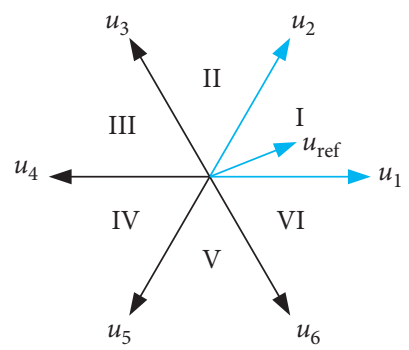

FIGURE 3: Schematic diagram of the basic voltage vector sector range.

resistance $0.15 \Omega$, rated torque $15 \mathrm{~N} \cdot \mathrm{m}$, rated speed $3000 \mathrm{r} / \mathrm{min}$, number of pole pairs 4 , and rotor inertia $0.00478 \mathrm{~kg} \cdot \mathrm{m}^{2}$.

5.1. The Steady-State Response Tests Results. At the beginning of the simulation, the motor runs at a rated speed of $3000 \mathrm{r} / \mathrm{min}$ and a rated torque of $15 \mathrm{~N} \cdot \mathrm{m}$.

Figure 5 is the $a$-phase current THD waveform of the motor under three different control methods. Figure 6 is the phase current waveform of $i_{a}$. Figure 7 shows the waveforms of $i_{d}$ ripple, $i_{q}$ ripple, and $T_{e}$ ripple of the motor at different speeds under three different control methods.

From Figures 5 and 6, it can be seen that the waveforms of RCBI-MPCC and ODC-MPCC are similar under the same conditions. As a result, RCBI-MPCC obtains steadystate control performance equivalent to ODC-MPCC. However, RCBI-MPCC does not need to predict the behavior of all voltage vectors when selecting voltage vectors, which reduces the calculation of the algorithm and reflects the superiority of the method. Since the selection range of the second voltage vector of RCBII-MPCC is not limited to the null voltage vector, its THD value is reduced by $26.01 \%$ compared to the traditional ODC-MPCC. Under this control, the $i_{d}$ ripple value, the $i_{q}$ ripple value, and the $T_{e}$ ripple value in the full speed range are all lower than RCBIMPCC. The simulation results show that RCBII-MPCC obtains better steady-state performance than RCBI-MPCC without significantly increasing the calculation burden.

5.2. The Dynamic Response Tests Results. In order to further compare the dynamic control performance of the proposed method, three control strategies are simulated for variable torque and variable speed control.

Figures 8-10, and show the simulation results of ODCMPCC, RCBI-MPCC, and RCBII-MPCC when performing variable torque control.

At the start of the simulation, the motor accelerates from still to rated speed $3000 \mathrm{r} / \mathrm{min}$, and then, at $0.2 \mathrm{~s}$, the load is suddenly increased to $15 \mathrm{~N} \cdot \mathrm{m}$.

From Figures 8-10, it is known that the $i_{d}, i_{q}, T_{e}$, and speed waveforms under the three control strategies are equivalent. The torque response time of the three control strategies is about $0.008 \mathrm{~s}$ when the motor is suddenly applied with a rated torque in $0.2 \mathrm{~s}$, indicating that their torque response speeds are equivalent. When the motor is under variable torque control, the speed under the three control 


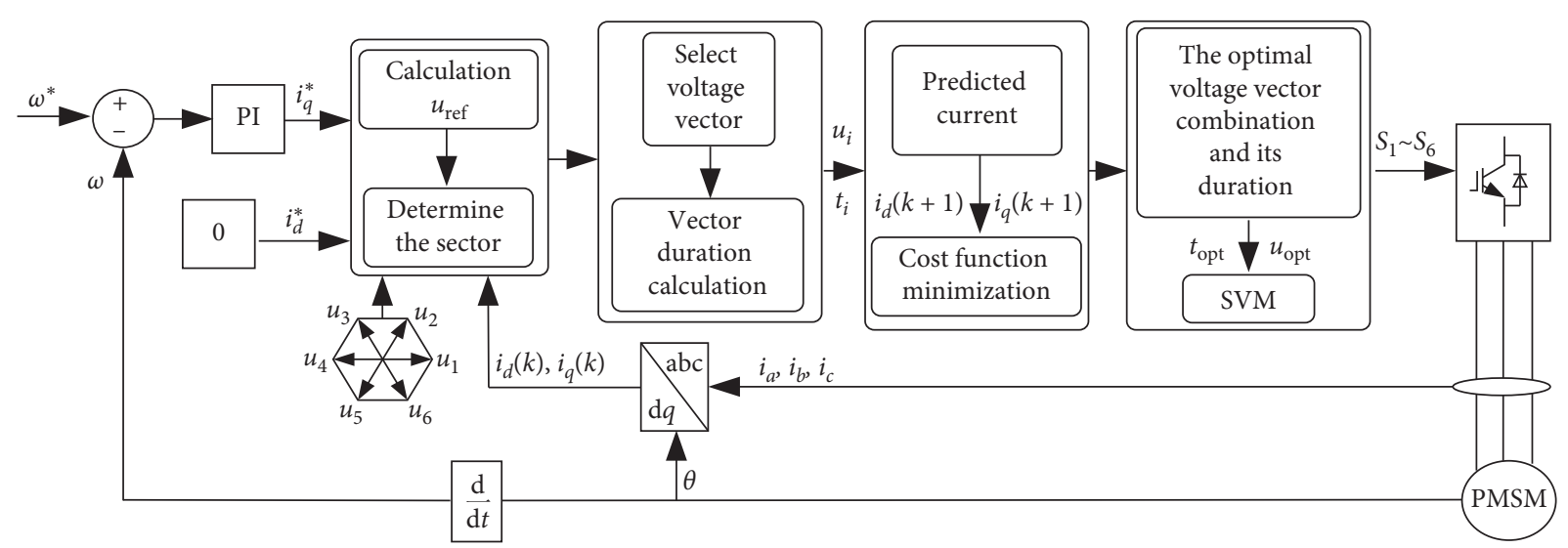

Figure 4: Control block diagram of the proposed MPCC.

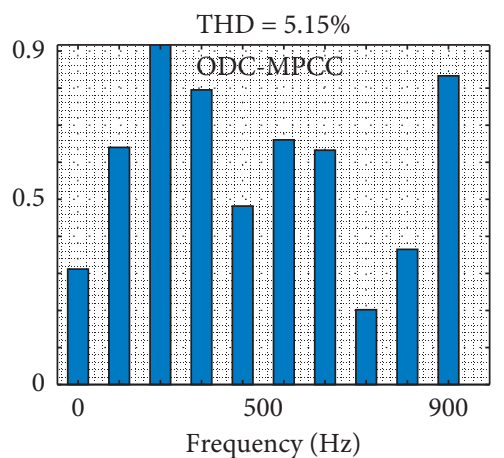

(a)

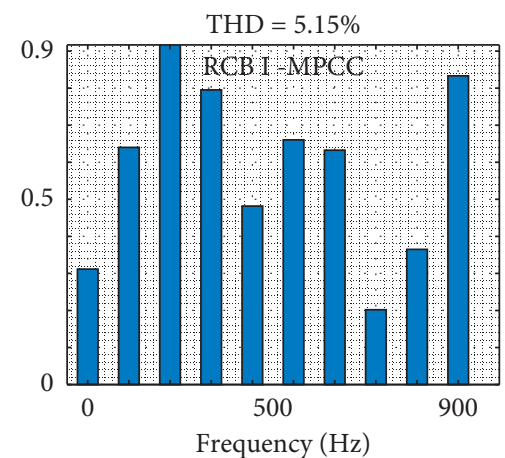

(b)

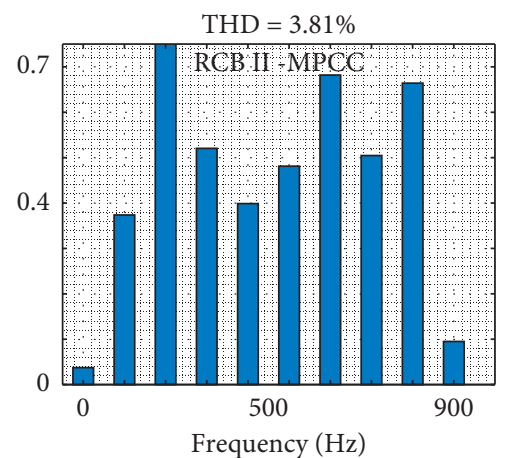

(c)

FIGURE 5: The harmonic spectrum of the $a$-phase stator current under the three methods at a rated speed of $3000 \mathrm{r} / \mathrm{min}$ and a rated load of $15 \mathrm{~N} \cdot \mathrm{m}$. (a) ODC-MPCC. (b) RCBI-MPCC. (c) RCBII-MPCC.

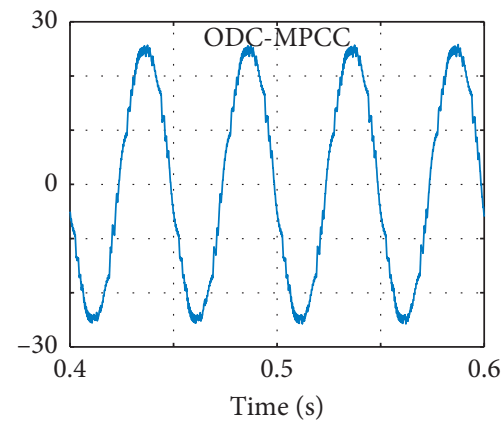

(a)

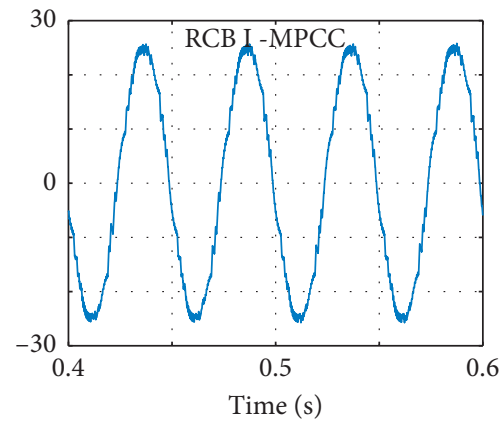

(b)

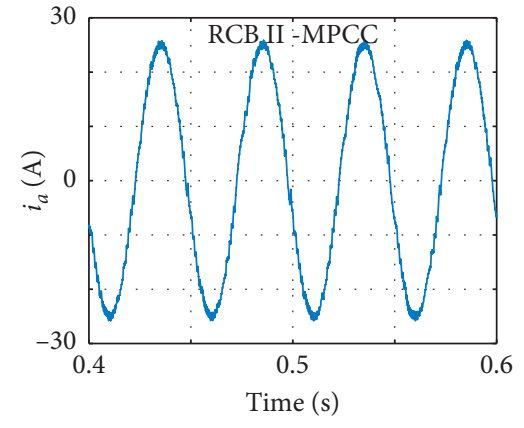

(c)

Figure 6: Three methods of $a$-phase stator current at rated speed $3000 \mathrm{r} / \mathrm{min}$ and rated load $15 \mathrm{~N} \cdot \mathrm{m}$. (a) ODC-MPCC. (b) RCBI-MPCC. (c) RCBII-MPCC.

strategies has a certain drop, but all quickly return to the rated speed after $0.19 \mathrm{~s}$, which shows that its antidisturbance ability is good. In summary, the three control strategies have equivalent dynamic performance when performing variable torque control. However, RCBI-MPCC avoids the large calculation burden caused by the traditional method of 6 predictions and simplifies the system's algorithm. RCBIIMPCC can obtain better steady-state performance without 


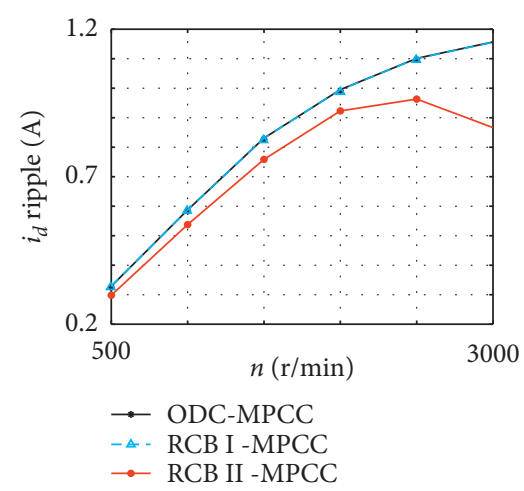

(a)

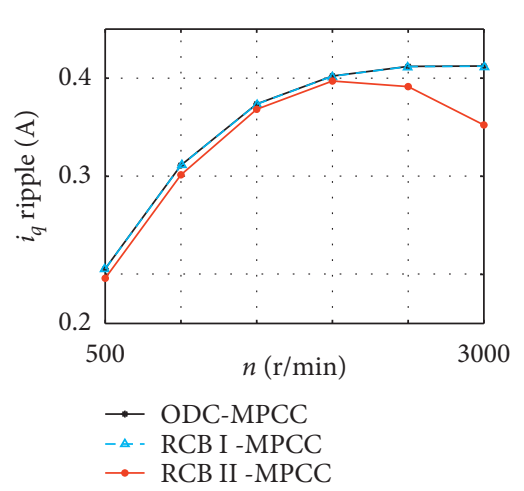

(b)

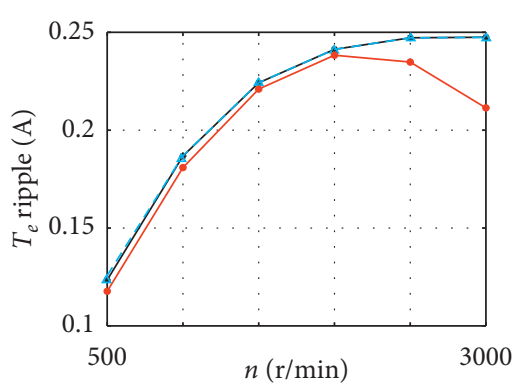

$\rightarrow$ ODC-MPCC

- - RCB I -MPCC

$\rightarrow$ RCB II -MPCC

(c)

Figure 7: Pulsating line chart of three methods at different speeds. (a) $i_{d}$ ripple. (b) $i_{q}$ ripple. (c) $T_{e}$ ripple.

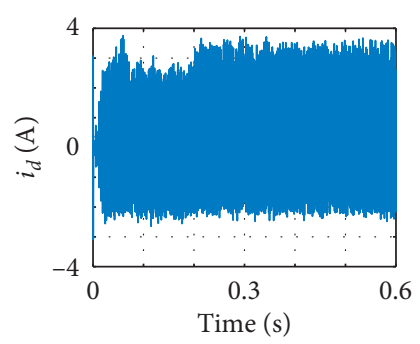

(a)

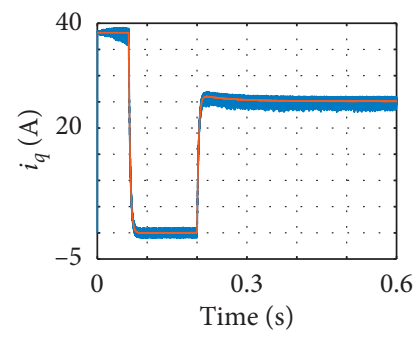

- Reference value

— Feedback value

(b)

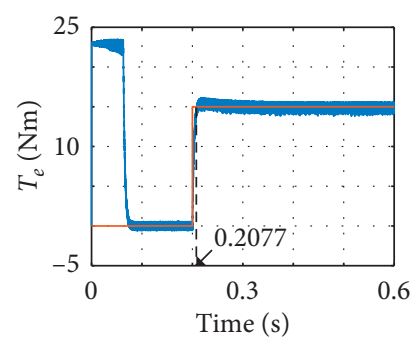

- Reference value — Feedback value

(c)

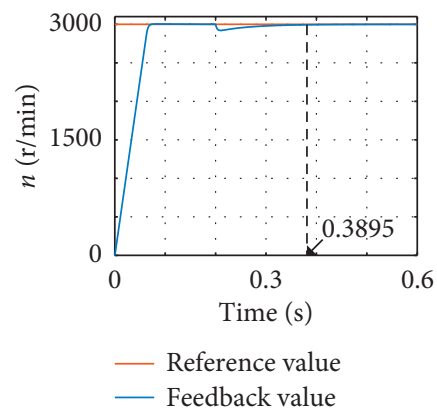

(d)

Figure 8: Simulation waveform of ODC-MPCC for variable torque control. (a) $d$-axis current. (b) $q$-axis current. (c) The waveform of torque. (d) The waveform of speed.

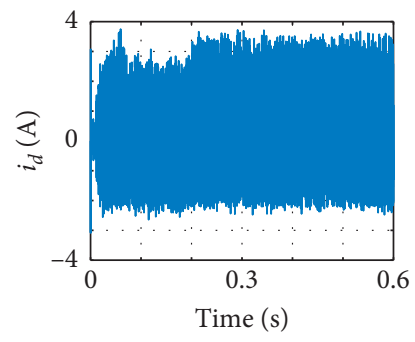

(a)

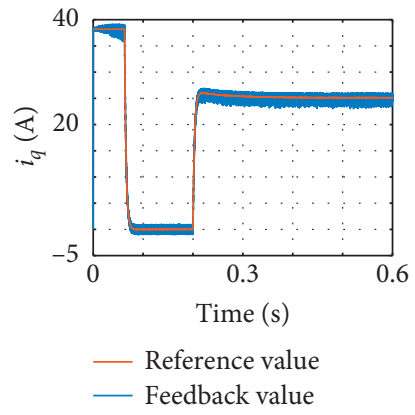

(b)

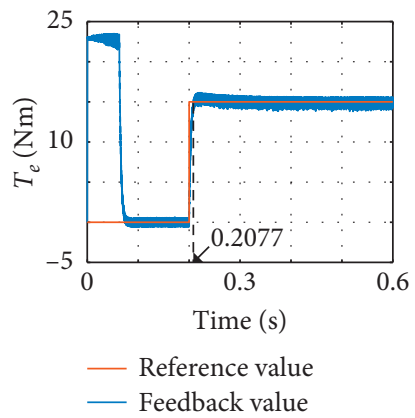

(c)

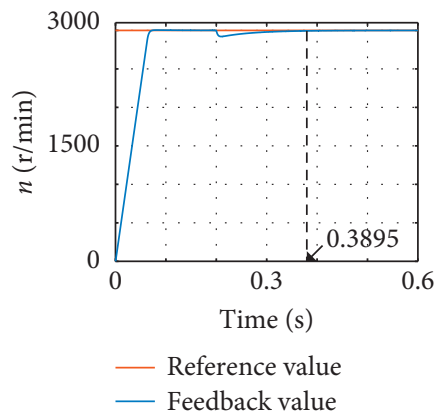

(d)

Figure 9: Simulation waveform of RCBI-MPCC for variable torque control. (a) $d$-axis current. (b) $q$-axis current. (c) The waveform of torque. (d) The waveform of speed.

significantly increasing the amount of calculation and has dynamic control performance comparable to RCBI-MPCC.

Figures 11-13, and show the simulation results of ODCMPCC, RCBI-MPCC, and RCBII-MPCC for variable speed control.

At the beginning of the simulation, the motor was running at a given speed of $1200 \mathrm{r} / \mathrm{min}$, and then, at $0.2 \mathrm{~s}$, the speed suddenly dropped to $600 \mathrm{r} / \mathrm{min}$.
According to Figures 11-13, the waveforms of $i_{d}, i_{q}, T_{e}$, and speed are similar under the three control strategies. The speed response time of the three control strategies is about $0.01 \mathrm{~s}$ when the motor speed drops suddenly in $0.2 \mathrm{~s}$. When the motor is under variable speed control, the torque under the three control strategies has a certain drop, but it quickly returns to the rated torque after $0.02 \mathrm{~s}$, which shows that the speed response performance is equivalent and the 


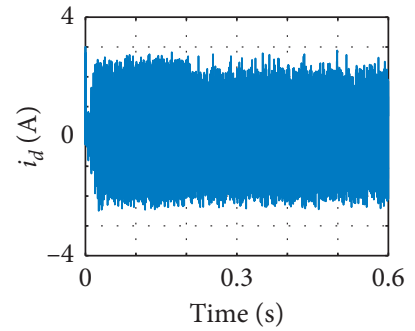

(a)

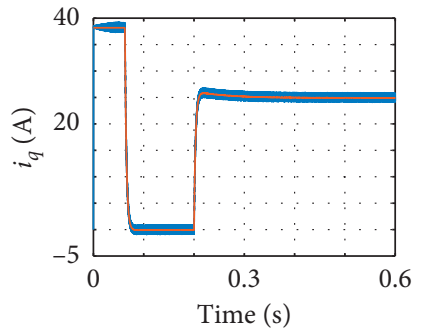

— Reference value

— Feedback value

(b)

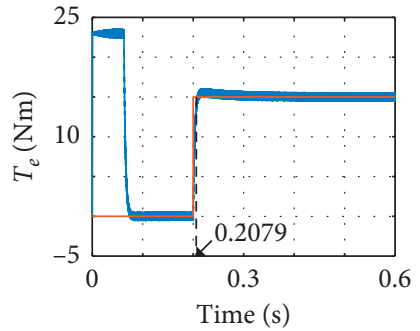

— Reference value

— Feedback value

(c)

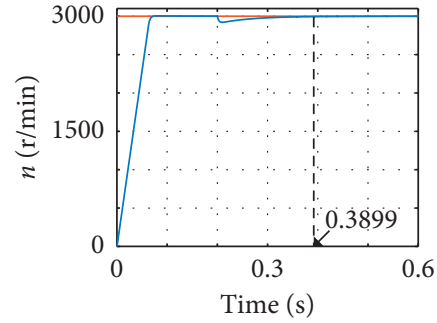

— Reference value

— Feedback value

(d)

Figure 10: Simulation waveform of RCBII-MPCC for variable torque control. (a) $d$-axis current. (b) $q$-axis current. (c) The waveform of torque. (d) The waveform of speed.

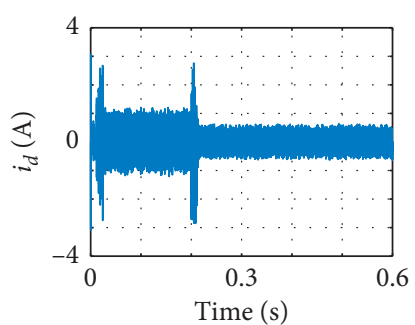

(a)

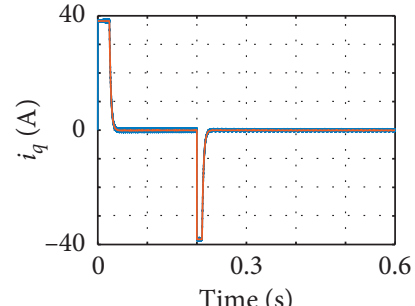

- Reference value

— Feedback value

(b)

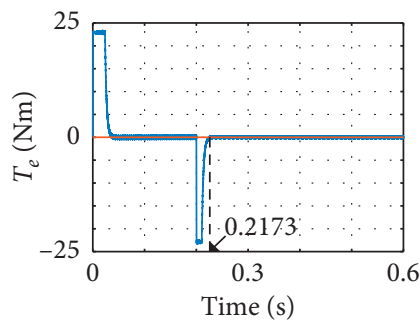

- Reference value

(c)

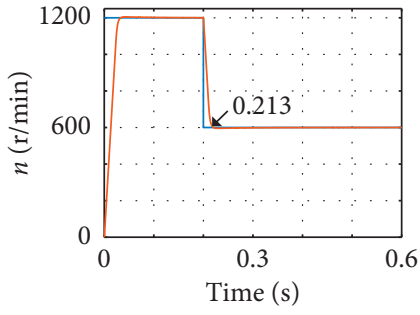

- Reference value

— Feedback value

(d)

FIGURE 11: Simulation waveform of ODC-MPCC for variable speed control. (a) $d$-axis current. (b) $q$-axis current. (c) The waveform of torque. (d) The waveform of speed.

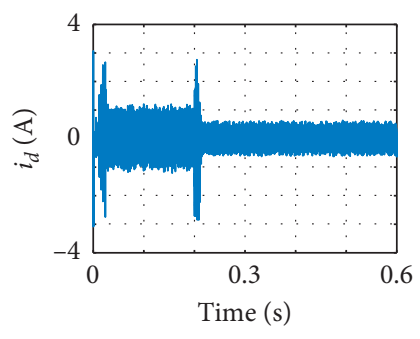

(a)

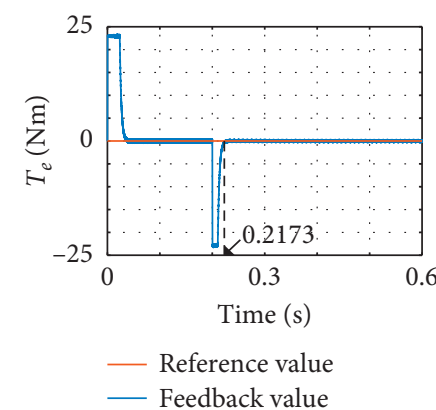

(c)

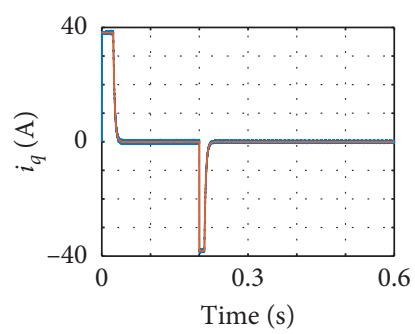

- Reference value

— Feedback value

(b)

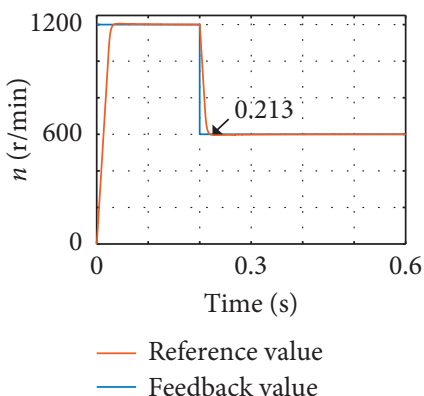

(d)

Figure 12: Simulation waveform of RCBI-MPCC for variable speed control. (a) $d$-axis current. (b) $q$-axis current. (c) The waveform of torque. (d) The waveform of speed. 


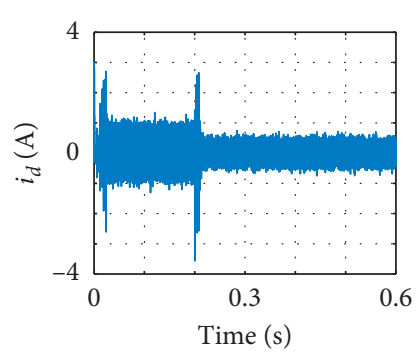

(a)

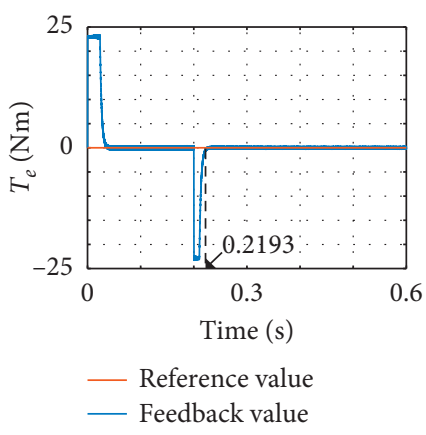

(c)

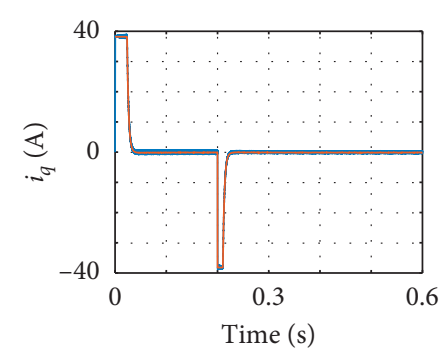

- Reference value

— Feedback value

(b)

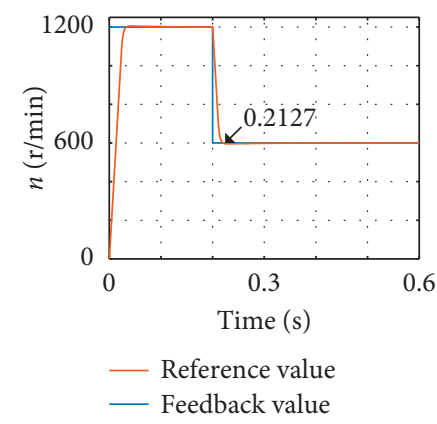

(d)

Figure 13: Simulation waveform of RCBII-MPCC for variable speed control. (a) $d$-axis current. (b) $q$-axis current. (c) The waveform of torque. (d) The waveform of speed.

antidisturbance ability is good. In summary, the three control strategies have considerable dynamic performance in variable speed control. The RCBI-MPCC reduces the number of candidate voltage vectors to 2 , which reduces the number of current predictions and reduces the calculation burden while ensuring the same control performance. RCBII-MPCC has a dynamic control performance equivalent to RCBI-MPCC while obtaining better steady-state performance.

\section{Conclusion}

This paper proposes an MPCC that reduces the computational burden. In the research, it is found that the optimal voltage vector selected by the deadbeat quadrature axis current control and the optimal voltage vector selected by the deadbeat current control are in the same sector and adjacent to each other. Using this law, the number of candidate voltage vectors is reduced from 6 to 2 .

After theoretical analysis and simulation verification, the conclusions are as follows:

(1) Compared with the traditional optimal duty cycle model predictive current control, the first control strategy reduces the computational burden of the algorithm by $66 \%$, and the second control strategy reduces the computational burden of the algorithm by $50 \%$.
(2) The dynamic response speed of the two proposed control strategies is equivalent to that of the traditional optimal duty cycle model predictive current control.

(3) The steady-state performance of the first proposed control strategy is equivalent to that of the traditional optimal duty cycle model predictive current control, while the second proposed control strategy has improved steady-state performance, and the THD value is reduced by $26.1 \%$.

\section{Data Availability}

The data used to support the findings of this study are included within the article.

\section{Conflicts of Interest}

The authors declare that there are no conflicts of interest regarding the publication of this paper.

\section{Acknowledgments}

This research was funded by the National Natural Science Foundation of China (Grant no. 51907061), Natural Science Foundation of Hunan Province (Grant no. 2019JJ50119), and National Engineering Laboratory of UHV Engineering Technology (Kunming, Guangzhou) (Grant no. NEL202008). 


\section{References}

[1] M. Wu, X. Sun, J. Zhu, G. Lei, and Y. Guo, "Improved model predictive torque control for PMSM drives based on duty cycle optimization," IEEE Transactions on Magnetics, vol. 57, no. 2, 2021.

[2] X. Sun, C. Hu, J. Zhu et al., "MPTC for PMSMs of EVs with multi-motor driven system considering optimal energy allocation," IEEE Transactions on Magnetics, vol. 55, no. 7, Article ID 8104306, 2019.

[3] X. Zhang, L. Zhang, and Y. Zhang, "Model predictive current control for PMSM drives with parameter robustness improvement," IEEE Transactions on Power Electronics, vol. 34, no. 2, pp. 1645-1657, 2019.

[4] H. T. Nguyen and J.-W. Jung, "Finite control set model predictive control to guarantee stability and robustness for surface-mounted PM synchronous motors," IEEE Transactions on Industrial Electronics, vol. 65, no. 11, pp. 8510-8519, 2018.

[5] A. A. Ahmed, B. K. Koh, and Y. I. Lee, "A comparison of finite control set and continuous control set model predictive control schemes for speed control of induction motors," IEEE Transactions on Industrial Informatics, vol. 14, no. 4, pp. 1334-1346, 2018.

[6] P. G. Carlet, F. Toso, A. Favato, and S. Bolognani, "A speed and current cascade continuous control set model predictive control architecture for synchronous motor drives," in Proceedings of the 2019 IEEE Energy Conversion Congress and Exposition (ECCE), pp. 5682-5688, Baltimore, MD, USA, September 2019.

[7] H. H. Choi, N. T.-T. Vu, and J.-W. Jung, "Digital implementation of an adaptive speed regulator for a PMSM," IEEE Transactions on Power Electronics, vol. 26, no. 1, pp. 3-8, 2011.

[8] S. Wang, C. Xia, X. Gu, and W. Chen, "A novel FCS-model predictive control algorithm with duty cycle optimization for surface-mounted PMSM," in Proceedings of the 7th IET International Conference on Power Electronics, Machines and Drives (PEMD 2014), pp. 1-6, Manchester, UK, April 2014.

[9] R. Mendez, D. Sbarbaro, and J. Espinoza, "High dynamic and static performance FCS-MPC strategy for static power converters," in Proceedings of the 2016 IEEE Energy Conversion Congress and Exposition (ECCE), pp. 1-7, Milwaukee, WI, USA, September 2016.

[10] J. Rodriguez, R. M. Kennel, J. R. Espinoza, M. Trincado, C. A. Silva, and C. A. Rojas, "High-performance control strategies for electrical drives: an experimental assessment," IEEE Transactions on Industrial Electronics, vol. 59, no. 2, pp. 812-820, 2012.

[11] W. Chen, S. Zeng, G. Zhang, T. Shi, and C. Xia, "A modified double vectors model predictive torque control of permanent magnet synchronous motor," IEEE Transactions on Power Electronics, vol. 34, no. 11, pp. 11419-11428, 2019.

[12] Y. Zhang and S. Gao, "Simultaneous optimization of voltage vector and duty cycle in model predictive torque control of PMSM drives," in Proceedings of the 2014 17th International Conference on Electrical Machines and Systems (ICEMS), pp. 3338-3344, Hangzhou, China, October 2014.

[13] M. Liu, S. Wang, and M. Zou, "Improved robust deadbeat predictive current control for PMSM using stator current and disturbance observation," in Proceedings of the 2019 IEEE International Symposium on Predictive Control of Electrical Drives and Power Electronics (PRECEDE), pp. 1-5, Quanzhou, China, May 2019.
[14] X. Zhang and B. Hou, "Double vectors model predictive torque control without weighting factor based on voltage tracking error," IEEE Transactions on Power Electronics, vol. 33, no. 3, pp. 2368-2380, 2018.

[15] Y. Zhang, S. Gao, and W. Xu, "An improved model predictive current control of permanent magnet synchronous motor drives," in Proceedings of the 2016 IEEE Applied Power Electronics Conference and Exposition (APEC), pp. 2868-2874, Long Beach, CA, USA, May 2016.

[16] Y. Zhang, L. Huang, D. Xu, J. Liu, and J. Jin, "Performance evaluation of two-vector-based model predictive current control of PMSM drives," Chinese Journal of Electrical Engineering, vol. 4, no. 2, pp. 65-81, 2018.

[17] Y. Xu, B. Zhang, and Q. Zhou, "A model predictive current control method of PMSM based on the simultaneous optimization of voltage vector and duty cycle," in Proceedings of the 2016 IEEE 8th International Power Electronics and Motion Control Conference (IPEMC-ECCE Asia), pp. 881-884, Hefei, China, May 2016.

[18] Y. Zhang and H. Yang, "Generalized two-vectors-based model predictive torque control of induction motor drives," in Proceedings of the 2014 IEEE Energy Conversion Congress and Exposition (ECCE), pp. 3570-3577, Pittsburgh, PA, USA, September 2014.

[19] Y. Xu, B. Zhang, and Q. Zhou, "PMSM double vector model predictive current control," Transactions of China Electrotechnical Society, vol. 32, no. 20, pp. 222-230, 2017.

[20] S. G. Petkar, K. Eshwar, and V. K. Thippiripati, "A modified model predictive current control of permanent magnet synchronous motor drive," IEEE Transactions on Industrial Electronics, vol. 68, no. 2, pp. 1025-1034, 2021.

[21] Y. Zhang, D. Xu, J. Liu, S. Gao, and W. Xu, "Performance improvement of model-predictive current control of permanent magnet synchronous motor drives," IEEE Transactions on Industry Applications, vol. 53, no. 4, pp. 3683-3695, 2017.

[22] X. Sun, M. Wu, G. Lei, Y. Guo, and J. Zhu, “An improved model predictive current control for PMSM drives based on current track circle," IEEE Transactions on Industrial Electronics, In press. 